\title{
Monocultivos e industrias extractivas en Guatemala: ¿Qué relación tienen la deficiencia institucional, la falta de investigación científica y los impactos ambientales?
}

\author{
Monoculture and extractive industries in Guatemala: What is the relationship between \\ institutional deficiency, lack of scientific research and environmental impacts? \\ Bárbara I. Escobar-Anleu
}

Escuela de Biología, Facultad de Ciencias Químicas y Farmacia, Universidad de San Carlos de Guatemala *Autor al que se dirige la correspondencia: bisa_e8a9@hotmail.com

Recibido: 27 de septiembre 2019 / Aceptado: 10 de febrero 2020

Resumen

G uatemala es considerado uno de los países megadiversos a nivel mundial. En conjunto, estos países albergan más del $70 \%$ de biodiversidad del planeta, en tan sólo alrededor del $10 \%$ de su territorio. Debido a la diversidad de recursos naturales, no resulta extraño que la economía del país dependa principalmente de estos. El ser una potencia mundial en biodiversidad podría representar múltiples ventajas y oportunidades, pero en realidad la degradación de los recursos naturales es histórica y persiste. Los monocultivos y la industria extractiva son ejemplos de actividades económicas que se han expandido en la región, contribuyendo al deterioro de los recursos naturales. Existe una paradoja, «la maldición de los recursos naturales», la cual se refiere a la situación en que países con muchos recursos naturales, suelen tener bajas tasas de crecimiento económico y una explicación es la debilidad institucional. El que existan deficiencias institucionales se relaciona con falta en cumplimiento de ley, una ineficiente regulación y manejo de recursos naturales y su consiguiente degradación. Si a esto agregamos que Guatemala invierte apenas el $0.029 \%$ del PIB en actividades de investigación, hallamos otra explicación a estas deficiencias, ya que las instituciones no tienen datos para tomar decisiones fundamentadas. Así se vincula el deterioro ambiental con las deficiencias institucionales, lo cual también se relaciona con poca investigación y reducido crecimiento económico. Aunque estos vínculos rara vez se resaltan, es necesario hacerlo para comprender y promover cambios dentro de un sistema explotador de recursos, que afecta la calidad de vida de los guatemaltecos.

Palabras clave: Ciencia, cultivos, divulgación científica, minería, recursos naturales.

Abstract

$\mathrm{G}$ uatemala is considered one of the megadiverse countries worldwide. Together, these countries contain more J than the $70 \%$ of the planet's biodiversity, in only about $10 \%$ of its territory. Due to the diversity of its natural resources, it is not surprising that the country's economy depends mainly on these. This great biodiversity could represent multiple advantages and opportunities, but the reality is that the degradation of natural resources is historical and persistent. Monocultures and extractive industry are examples of activities that have expanded in the region, contributing to the deterioration of natural resources. There is a paradox, "the curse of natural resources", which refers to the situation in which countries with many natural resources tend to have low rates of economic growth and an explanation for this is institutional weakness. The existence of institutional deficiencies is related to lack of compliance with the law, inefficient regulation and management of natural resources and their consequent degradation. If we add to this that Guatemala invests only $0.029 \%$ of GDP in research activities, we find another explanation for these deficiencies, since the institutions do not have data to make informed decisions. This links environmental deterioration with institutional deficiencies, which is also related to little research and reduced economic growth. Although these links are rarely highlighted, it is necessary to do so in order to understand and promote changes within an exploitative resource system that affects the life quality of all Guatemalans.

Key Word: Crops, mining, natural resources, science, science communication.

Este trabajo fue revisado por el jurado calificador del Quinto Concurso de Ensayo Científico Digi-2017, obteniendo el segundo lugar para el área Ciencia y Tecnología. 


\section{Introducción}

La Constitución Política de la República de Guatemala declara en el artículo 64 que es "de interés nacional la conservación, protección y mejoramiento del patrimonio natural de la nación" (1985). Además, establece como "obligación del Estado orientar la economía nacional para lograr la utilización de los recursos naturales y el potencial humano, para incrementar la riqueza y tratar de lograr el pleno empleo y la equitativa distribución del ingreso nacional" (Constitución Política de la República de Guatemala, 1985). Sin embargo, existen actividades que desde una perspectiva mercantil podrían parecer rentables y/o eficientes pero los impactos que tienen sobre los recursos naturales son severos e irreversibles. Este es el caso de actividades como las de la industria extractiva y los monocultivos. Al respecto, existe información, pero esta es principalmente de los impactos sociales. ¿Qué pasa con los impactos ambientales? ¿Existe suficiente investigación y respaldo científico en estos temas? ¿Qué relación tienen la deficiencia institucional y la escasa investigación científica con los impactos ambientales de estas industrias?

Para documentar los impactos de estas industrias en Guatemala, es necesario tener datos e información fidedigna, la cual suele ser escasa o de difícil acceso. A continuación, se presentan algunos datos relacionados con estos temas, con el objetivo de evidenciar la relación entre estos temas y así comprender esta compleja problemática desde una perspectiva más integral. los cuales se pretende abordar el tema del impacto ambiental de estas actividades y la importancia de la investigación científica con una de las limitantes que tienen como denominador común: la deficiencia institucional. Este enfoque se utilizará.

\section{Contenido}

A nivel mundial, Guatemala es el cuarto país más vulnerable en términos de desastres naturales y el noveno en términos de riesgos por el cambio climático (Organización de las Naciones Unidas para la Educación, la Ciencia y la Cultura [Unesco], 2017). Los economistas sostienen que el desarrollo depende de la medida en que se utilicen los recursos disponibles en el territorio del país (Instituto de Agricultura, Recursos Naturales y Ambiente de la Universidad Rafael Landívar [Iarna-URL], 2012).
Si se considera que la economía guatemalteca depende de sus recursos naturales, al menos en un $70 \%$ (Iarna-URL, 2012), su deterioro tiene un particular agravante a nivel nacional. En la década de los 60 y 70, la principal causa de deforestación era la presión poblacional y la pobreza (Universidad del Valle de Guatemala, Instituto Nacional de Bosques, Consejo Nacional de Áreas Protegidas, \& Universidad Rafael Landívar, 2011) pero actualmente tiene su origen en actividades como la ganadería extensiva, la agricultura vinculada a monocultivos extensivos -como la palma africana-, los incendios forestales y las combinaciones entre éstas (Iarna-URL, 2012).

Por otro lado, por definición, las actividades extractivas no son sostenibles ya que, al extraer los recursos del subsuelo, los yacimientos se agotan y no se restablecen en el corto plazo (Instituto Centroamericano de Estudios Fiscales [Icefi], 2014). Ambas actividades ejemplifican lo que en literatura económica se ha denominado la "maldición de los recursos naturales" (Sachs \& Warner, 1995, 2001) que se refiere a la paradójica situación en que países con muchos recursos naturales, tienden a tener bajas tasas de crecimiento económico.

En el 2011 el tanque de pensamiento del sector privado Fundación para el Desarrollo de Guatemala (Fundesa) realizó un estudio donde identificó 25 conglomerados productivos con gran potencial de crecimiento, entre los cuales incluyó la agricultura (asociada a cultivo de monocultivos de palma y azúcar) y las industrias extractivas (Unesco, 2017). Sin embargo, a nivel regional, en el 2016 el producto interno bruto (PIB) de América Latina y el Caribe disminuyó un $11 \%$, tasa negativa que representa la continuación de un proceso de desaceleración de la actividad económica que existe desde el 2011 (Comisión Económica para América Latina y el Caribe [Cepal], 2017). Una de las explicaciones que da la economía política a esta "maldición" es que en países con instituciones con un marco legal inadecuado e instituciones débiles afectan el desempeño de su crecimiento, mientras que instituciones con marcos adecuados impulsan altas tasas de crecimiento (Boschini, Pettersson, \& Roine, 2007; Mehlum, Moene, \& Torvik, 2006).

Por otro lado, la deficiencia institucional también está vinculada con la falta de investigación científica que existe en el país ya que en Guatemala no existe una cultura institucional de seguimiento y evaluación periódica, principalmente por falta de fondos. Aunque la industria en el país tiene cierta disponibilidad de recursos económicos, no realiza investigación ya que 
no existen incentivos ni otros instrumentos políticos para que lo hagan (Unesco, 2017). Guatemala invierte el $0.029 \%$ del PIB en actividades de investigación y desarrollo experimental. Este porcentaje es 14 veces menor que el promedio de inversión de África Subsahariana, 25 veces menos que el de Latinoamérica y el Caribe, 85 veces menor que el de Europa Occidental y casi 200 veces menor que lo que invierte Corea o Israel (Unesco, 2017). La poca investigación que se genera en el país también puede verse reflejada en el número de artículos que se producen. Actualmente, el valor de artículos es de 11 por millón de habitantes. Para tener una comparación, la publicación de artículos científicos en Chile es 45 veces mayor por millón de habitantes. Además, alrededor del $90 \%$ de artículos de Guatemala se publican en cooperación con otros países (Unesco, 2017). Es de esta forma que se puede vincular el deterioro ambiental de los recursos naturales causado por estas actividades con las deficiencias institucionales, que están a su vez directamente relacionadas con la falta de investigación y la disminución en el crecimiento económico del país.

Aunque pocas veces se ve desde esta perspectiva, es necesario resaltar este vínculo para poder trabajar en las deficiencias existentes, que resultan teniendo un impacto en los recursos naturales y así, en la población guatemalteca. Resulta interesante cómo Guatemala se encuentra (junto con Argentina, Bolivia, Brasil, Colombia, Ecuador, El Salvador, Guyana, Haití, Honduras, Nicaragua, Paraguay, Perú y Venezuela) entre las naciones con valores negativos, tanto en su eficiencia gubernamental, como en su estabilidad política (Lemarchand, 2013). Existen estudios que evidencian cómo la productividad científica y la influencia sobre ella, se relaciona con características como la gobernanza, el crecimiento económico y la distribución de ingresos para desarrollo humano (Lemarchand, 2015, 2016).

Aunque hace falta investigación científica enfocada hacia el impacto ambiental de estas dos industrias, los datos que se tienen no son muy alentadores. En el caso de los monocultivos, revertir la trayectoria negativa que tienen las dinámicas del uso del suelo es un gran desafío. A largo plazo, restaurar y restituir recursos del bosque contribuirá significativamente en la estabilidad ambiental y económica de Guatemala. Sin embargo, en el caso de la caña de azúcar las prácticas de este monocultivo requieren homogeneización de las áreas de cultivo. Este proceso suele empezar con la eliminación de árboles y otra cobertura vegetal, relleno de barrancos para incrementar áreas mecanizables y construcción de canales para desviar los cauces de los ríos para los canales de riego (Iarna-URL, 2012).

En el caso de la palma africana, debido a que la productividad promedio es de 25 años, no existe rotación en el monocultivo y así el suelo se desgasta y pierde su fertilidad. Para compensar esto, la industria enriquece artificialmente el suelo a través de agroquímicos, de los cuales se requieren cada vez cantidades mayores y empobrecen aún más el suelo. Los monocultivos evidentemente reducen la diversidad vegetal, la cual a su vez disminuye la diversidad animal debido a que anula o interrumpe la dinámica de los ecosistemas (Hernández, 2013). Esto se ha demostrado en estudios en monocultivos de palma africana en el país, los cuales han determinado que éstos son una seria amenaza para la diversidad de aves, debido a que únicamente el 11 $\%$ de especies y el $5 \%$ del total de capturas durante el estudio, se realizaron en cultivos de palma del área de estudio (Cajas-Castillo, Cóbar-Carranza, Ávila-Santa, Kraker-Castañeda, \& Quiñónez-Guzmán, 2015).

Las especies de fauna cumplen un papel fundamental controlando plagas, pero al reducir las especies vegetales, muchas especies que antes se alimentaban de ellas desaparecen y así, también sus depredadores. Uno de los resultados de esta alteración en la dinámica ecológica, es que las plagas se propagan con mayor facilidad, dañando así los cultivos (Hernández, 2013). Otro aspecto de riesgo al ambiente con los monocultivos es que, con su acelerada expansión, las pocas áreas naturales protegidas van quedando aisladas y esto interrumpe la conectividad y reduce la diversidad (Edwards et al., 2010). Para el 2006, la extensión registrada de palma africana era de 53,908 ha, incrementando en un $73.47 \%$ (39,607 ha) para el 2010, cuando ascendió a 93,515 ha (Duarte, Juárez, Pérez, \& Gálvez, 2010). Surge la pregunta entonces ise está haciendo lo necesario para que este cultivo, considerado tan eficiente, también considere mitigar o reducir los impactos ecológicos que pueda tener en un área?

La información ambiental que tenemos indica que no. Los problemas generados a partir del monocultivo de palma africana han contribuido en la ruptura y el daño a estructuras, no sólo ecosistémicas, sino también socioambientales y nutricionales en territorios rurales (Hernández, 2013). Para reafirmar y seguir vinculando la importancia de la investigación científica y la institucionalidad con estas industrias, existen referencias documentadas, como en el caso de Colombia, donde los mismos productores de palma han reconocido que no saben de conservación ni de criterios ambientales 
y que establecer límites y control de la expansión le corresponde a instituciones ambientales y agrícolas (Hernández, 2013). Si bien no tenemos declaraciones como estas en Guatemala, sí sabemos que los vínculos entre el sector empresarial y el sector académico son escasos y, aunque el $50 \%$ de los investigadores debería estar realizando investigación e innovación dentro del sector productivo del país, esto no es así (Unesco, 2017), conllevando esto un riesgo similar al caso de Colombia.

En el caso de la industria extractiva, los impactos al capital natural en Guatemala se dan en un contexto muy particular, ya que el país tiene la tasa más alta de extracción de materiales por unidad de área (8.3TM/ ha/año). Esta tasa sobrepasa el promedio de países con indicadores económicos similares (4.8 TM/ha/año) (Iarna-URL, 2012). A la industria extractiva, junto con las actividades agrícolas y ganaderas, se les atribuye, por lo menos, un $15 \%$ del sobreuso existente en el territorio nacional (Icefi, 2014).

El fenómeno de rápida expansión de industrias extractivas ha sido especialmente agudo en Centroamérica, provocando conflictos ambientales y sociales (Icefi, 2014). Aunque las implicaciones ambientales de la minería son de diversa índole y varían de intensidad a lo largo de las distintas etapas del proyecto, pueden agruparse por sus efectos en el agua, aire, suelo y ecosistemas (Iarna-URL, 2012).

En el caso del recurso hídrico, es significativamente afectado en calidad y cantidad. Los cuatro aspectos que generan un impacto significativo son: primero, el drenaje ácido de mina y lixiviados; segundo, la erosión de suelos y desechos en aguas superficiales; tercero, los impactos causados por embalses, desechos rocosos y lixiviación en pilas y botadores y cuarto, los impactos por el desaguado de la mina. En el caso del aire, es afectado durante todas las etapas del proyecto ya que se moviliza una gran cantidad de materiales. Incluso cuando no se moviliza, las pilas o depósitos de desechos dispersan partículas al ambiente. Durante las operaciones mineras, las mayores fuentes contaminantes para el aire son: el material en partículas que se transportan como resultado de actividades mecánicas (como la excavación), las emisiones gaseosas de los escapes de fuentes móviles (vehículos, etc.) y las emisiones de gases provenientes de la quema de combustibles en fuentes estacionarias (Icefi, 2014).

En el caso del suelo, las actividades agrícolas cercanas a este tipo de proyectos pueden verse afectadas, principalmente, por la contaminación que se esparce a través de la escorrentía o el agua para riego. Además, las actividades mineras modifican diariamente el paisaje cercano a través de la remoción de materiales que antes no estaban perturbados (Environmental Protection Agency [EPA], 2009). La erosión causada por la minería, también puede resultar en el aumento de carga de sedimentos en drenajes y aguas superficiales, así como en la contaminación de suelos por los derrames y el vertido de materiales tóxicos (Icefi, 2014).

En el caso de los ecosistemas, la pérdida de hábitat es a través de daños directos e indirectos, así como a corto y largo plazo. A corto plazo son la perturbación, remoción y redistribución de superficie en el lugar de la mina. Sin embargo, hay impactos de mayor alcance y largo plazo como la eliminación o desplazamiento de especies en áreas excavadas y depósitos de desechos. A escala terrestre, algunas especies se van del área, pero existen otras con menor capacidad de movilización (invertebrados y vertebrados pequeños) que son los más afectados, mientras que los impactos a los ecosistemas acuáticos también son recurrentes en los proyectos de minería, sobre todo en países con debilidades en el ámbito legal e institucional, como el caso de Guatemala (Icefi, 2014).

Esto ha sido evidenciado en casos como el de la mina Marlin en San Marcos. En este caso, el agua almacenada en los embalses de cola excede los lineamientos establecidos por la Corporación Financiera Internacional (IFC) para $\mathrm{pH}$, cianuro, cobre y mercurio siendo las concentraciones de estos últimos de tres, 10 y 20 veces mayores, respectivamente. El tratamiento de agua no resuelve la filtración de contaminantes y la limitada información sobre calidad de agua no ha permitido establecer si se está filtrando corriente abajo (Icefi, 2014).

Otro aspecto ambiental relacionado con las actividades extractivas en Guatemala, al cual se le ha prestado poca atención, es la relación entre el grado de fragilidad ambiental y el conflicto. Los municipios donde hay actividades mineras y con conflictos reportados tienen, en promedio, 44.1 mayor deforestación y 24.7 $\%$ más territorio en cabeceras de cuenca que el promedio nacional (Instituto Regional de Altos Estudios Políticos [Iralep], 2010). Esto refleja que las actividades extractivas coinciden con áreas que ya tienen fragilidad ambiental lo cual es preocupante al considerar que el $38 \%$ de las últimas extensiones de bosques primarios mundiales, están en riesgo por la expansión de actividades mineras (Carrere, 2004).

Aún más preocupante resulta que, si se mantiene la trayectoria actual, la minería en Guatemala se carac- 
terizará por una presión incremental, poca capacidad de respuesta de un marco normativo y otras amenazas cuyo factor en común es la fragilidad del marco legal e institucional en materia ambiental (Icefi, 2014).

A lo largo de este documento se ha pretendido vincular la deficiencia institucional con la falta de investigación y los impactos que esto tiene en los recursos naturales, específicamente en las actividades extractivas y los monocultivos en el país. A continuación, se fundamentan algunas posibles acciones o medidas que podrían contribuir a la conservación de los recursos naturales, mediante la investigación científico y el fortalecimiento institucional en las industrias de monocultivos, así como en las extractivas. Es un hecho que se necesita hacer más investigación, principalmente en estas industrias, las cuales también han sido priorizadas por el Plan Nacional de Desarrollo K'atún (Consejo Nacional de Desarrollo Urbano y Rural [Conadur], 2014). El fundamento de priorizar la agricultura es que en ella se emplea una tercera parte de la fuerza laboral de Guatemala, por lo que la importancia de este sector se mantendrá y para ello se propone promover la diversificación productiva (lo cual se relaciona con limitar la expansión de los monocultivos), fortalecer las instituciones y fomentar el desarrollo rural mediante inversión en investigación y desarrollo agrícola (Unesco, 2017).

Debido a que la mitad de la población trabaja en el sector agropecuario, la concentración nacional de tierras se limita a un $2 \%$ de personas que controlan hasta el $72 \%$ del total de tierras cultivables (Gauster \& Isakson, 2007; Klepek, 2012). Esta desigualdad restringe de gran manera el desarrollo sostenible y equitativo del país, imponiendo restricciones para difundir propuestas, nuevas tecnologías y mecanismos novedosos para producción agropecuaria, que permita conservar la biodiversidad y proteger el medio ambiente. Nuevamente aquí, entran a jugar un papel importante las instituciones de investigación del país que realizan extensión y propuestas en estas áreas (Unesco, 2017).

Por otro lado, las actividades extractivas también son prioritarias en este plan debido a que, a pesar de que son una fuente limitada en la generación de trabajo y tienen importantes impactos negativos hacia el ambiente, constituyen una fuente de recursos que se podría aprovechar mejor (Unesco, 2017). ¿Cómo sería esto posible? Si bien no existen actividades extractivas sostenibles, ciertos autores sugieren algunas alternativas, como asumir una transformación de una forma de capital a otra. En este sentido, una de las propuestas es gestionar adecuadamente todas las formas de capital.
En la minería, un ejemplo podría ser que con la reducción de activos del subsuelo se busquen mecanismos de compensación mediante otros activos naturales, como la inversión para eliminar externalidades negativas bajo el principio de "quien contamina o usa, paga" (Icefi, 2014).

El Plan Nacional de Desarrollo K'atún propone gestionar subvenciones y regulación ambiental, mejorar la gobernanza, fortalecer regulaciones y localizar las actividades en función del mejor uso de suelo (Conadur, 2014; Unesco, 2017). Todas estas propuestas, pueden fundamentarse a través de investigación científica. Para que las actividades de investigación e innovación puedan empezar a tener un impacto visible y considerable en la economía del país, el tamaño de la comunidad científica nacional debería ser, por lo menos, 45 veces más grande que en la actualidad (Unesco, 2017).

Una forma de lograr esto es integrar esfuerzos institucionales, lo cual ha sido difícil para los gobiernos debido a que las estructuras de las instituciones suelen ser inadecuadas (a nivel de departamento, falta de continuidad, con agendas individuales y no integrales) para abordar de manera transversal temas como la investigación y la innovación tecnológica. Hasta ahora, las políticas en ciencia, tecnología e investigación han sido gestionadas por distintos ministerios, universidades y centros investigativos, pero con poca o nula interacción e integración entre ellos. Esta ha sido una limitante importante ya que para que las actividades de investigación sean eficaces y eficientes, requieren coherencia y coordinación entre distintos programas, departamentos y políticas de los gobiernos. La investigación e innovación tecnológica deben ser transversales, ya que concierne a distintas instituciones y ministerios: ciencia y tecnología, educación superior, salud, agricultura, energía y minas, medio ambiente, planificación, etc. (Unesco, 2017).

Adoptar un enfoque coherente no sólo implica coordinar diversas medidas políticas dictadas por el conjunto básico, como las de las universidades y las empresas, sino que también implica su interacción con otras políticas que persigan otros objetivos, tales como políticas fiscales y leyes y reglamentos de competencia que sirvan dentro del marco de innovación e investigación (Organisation for Economic Cooperation and Development [OECD], 2010).

Respecto a la actividad extractiva en Guatemala, existen desafíos que deben enfrentarse de manera inmediata y paralela. Esto es debido a que son retos que dependen uno del otro y si se abordaran de manera 
aislada, difícilmente se logrará un resultado útil. Los desafíos de interés en este caso, se relacionan con la necesidad de acciones a corto y mediano plazo, con el fin de mejorar mecanismos regulatorios y de distribución del estado, así como ampliar el debate público y la información sobre la minería para planificar a largo plazo (Icefi, 2014).

Para esto es necesario mejorar las capacidades técnicas y fortalecer las instituciones encargadas de regular la industria extractiva, así como implementar una política fiscal en la cual la industria contribuya en justa dimensión a la generación de la riqueza que obtiene, para aumentar así la efectividad en el ingreso, la distribución y el gasto de recursos. Es necesario que el Estado pueda captar suficientes ingresos de estos recursos para que puedan ser reinvertidos en otras formas de capital, como la institucionalidad ambiental. Esto, es necesario para garantizar monitoreos e investigación de los impactos en los recursos naturales y así fiscalizar que las normativas ambientales se estén cumpliendo (Icefi, 2014).

Respecto a ampliar el debate público y la información sobre la minería, la base para lograrlo es generar suficiente información, que sea fiable, de buena calidad y que permita definir costos y beneficios reales de esta industria (Icefi, 2014). Estos desafíos, nuevamente dejan en evidencia la necesidad de realizar investigación científica para poder fundamentar políticas y otros de control, regulación y monitoreo. El desarrollo de la ciencia y la tecnología es fundamental para cualquier país. En Guatemala, el desarrollo científico requiere estructuras y mecanismos que permitan su participación en investigación y aplicación de la información generada. Dos de las áreas prioritarias para investigar son la biodiversidad y la agroalimentación, ya que son de interés científico, pero también tienen proyección social a nivel nacional y regional (Conadur, 2014; Unesco, 2017).

Tanto los monocultivos, como las industrias extractivas en Guatemala están vinculados con estas áreas prioritarias para desarrollar investigación científica y tecnología. La ciencia no es algo aislado de la sociedad, es necesaria para que esta prospere. A través de promover, financiar y priorizar la investigación científica en Guatemala estaremos fortaleciendo las políticas, propuestas y generando mecanismos que permitan el desarrollo nacional.

Es importante invertir en investigación, tanto en el ámbito público como el privado, ya que es de esta manera que se puede obtener información fidedigna, no sólo de los impactos que producen las industrias, sino también de las medidas que se pueden proponer a nivel institucional para su fortalecimiento. La necesidad de comprender los beneficios que tiene la investigación en la vida cotidiana puede resumirse de la siguiente manera: promover la ciencia e investigación permite fortalecer las instituciones con información, datos reales y útiles. Este fortalecimiento a su vez permitirá generar propuestas que permitan la toma de decisiones fundamentadas, equilibrando el desarrollo económico del país mientras se conserva la biodiversidad y los recursos naturales y esto se relaciona con la salud y un futuro más digno para los guatemaltecos.

\section{Conclusiones}

Históricamente, los recursos naturales de Guatemala no han sido manejados adecuadamente y conforme las actividades humanas, agrícolas e industriales se expanden, es importante tener información del antes, durante y después de éstas. Las empresas e instituciones, tanto académicas, como gubernativas y no gubernativas deben promover, facilitar y realizar investigación para que las decisiones que se tomen en el manejo y aprovechamiento de los recursos naturales realmente sean las apropiadas y que así el desarrollo no sea visto únicamente desde una perspectiva económica, sino priorizando una calidad de vida más digna para las y los guatemaltecos. Debemos aprender a utilizar la ciencia y la información que nos permite generar, por lo que termino con una apropiada reflexión del científico Albert Einsten: “¿Por qué esta magnífica tecnología científica, que ahorra trabajo y nos hace la vida más fácil, nos aporta tan poca felicidad? La respuesta es muy sencilla: es simplemente porque aún no hemos aprendido a usarla con tino".

\section{Referencias}

Boschini, A., Pettersson, J., \& Roine, J. (2007). Resource curse or not: A question of appropriability. Scandinavian Journal of Economics, 109(3), 593-617. https://doi.org/10.1111/ j.1467-9442.2007.00509.x

Cajas-Castillo, J. O., Cóbar-Carranza, A. J., Ávila-Santa, R. C., Kraker-Castañeda, C., \& Quiñónez-Guzmán, J. (2015). Diversidad de aves de sotobosque en bosques tropicales, áreas de regeneración natural y cultivos de palma africana en humedales 
del Lago de Izabal, Guatemala. Ornitología Neotropical, 26(1), 1-12.

Carrere, R. (2004). Minería, impactos sociales y ambientales. Montevideo, Uruguay: Movimiento Mundial por los Bosques Tropicales.

Comisión Económica para América Latina y el Caribe. (2017). Panorama Económico y Social de la Comunidad de Estados Latinoamericanos y Caribeños, 2016. Santiago, Chile: Autor

Consejo Nacional de Desarrollo Urbano y Rural. (2014). Plan Nacional de Desarrollo K'atún: Nuestra Guatemala 2032. Guatemala: Autor.

Constitución Política de la República de Guatemala: Reformada por el Acuerdo Legislativo 18-93, art. 119, inciso c. 4 , ibid., art. 121, inciso e. (17 de noviembre de 1993)

Duarte, C., Juárez, M., Pérez, I., \& Gálvez, J. (2012). Análisis de la dinámica de expansión del cultivo de la palma africana en Guatemala: Un enfoque cartográfico. En Perfil Ambiental de Guatemala 2010-2012 (pp. 340-362). Guatemala: Universidad Rafael Landívar,Instituto de Agricultura, Recursos Naturales y Ambiente.

Edwards, D. P., Hodgson, J. A., Hamer, K. C., Mitchell, S. L., Ahmad, A. H., Cornell, S. J., \& Wilcove, D. S. (2010). Wildlife-friendly oil palm plantations fail to protect biodiversity effectively. Conservation Letters, 3(4), 236-242. https://doi. org/10.1111/j.1755-263X.2010.00107.x

Environmental Protection Agency. (2009). Profile of the Metal Mining Industry. United States: Autor.

Gauster, S., \& Isakson, R. (2007). Eliminating market distortions, perpetuating rural inequality: An evaluation of market-assisted and reform in Guatemala. Third World Quarterly, 28(8), 1519-1536. https://doi.org/10.1080/01436590701637375

Hernández, S. (2013). Consecuencias negativas de la palma africana en Guatemala. ConCiencia, 3, 78-87.

Instituto de Agricultura, Recursos Naturales y Ambiente de la Universidad Rafael Landívar. (2012). Perfil Ambiental de Guatemala 2010-2012. Vulnerabilidad local y creciente construcción de riesgo. Guatemala: Autor.

Instituto Centroamericano de Estudios Fiscales. (2014). La minería en Guatemala: Realidad y desafios frente a la democracia y el desarrollo. Guate- mala: Autor.

Instituto Regional de Altos Estudios Políticos. (2010). Comprendiendo el conflicto sobre minería en Guatemala. Guatemala: Autor.

Klepek, J. (2012). Selling Guatemala's next Green Revolution: Agricultural modernization and the politics of GM maize regulation. International Journal of Agricultural Sustainability, 10(2), 117-134. https://doi.org/10.1080/14735903.201 2.641326

Lemarchand, G. A. (2013). Science, Technology and Innovation Information-Platform (STIIP) for $\mathrm{Na}$ mibia: A resource for the formulation, monitoring and evaluation of research and innovation policies. Paris: Organización de las Naciones Unidas para la Educación, la Ciencia y la Cultura.

Lemarchand, G. A. (2015). Latin America. En UNESCO science report: Towards 2030 (Cap. 7, pp. 175-209). Paris: Organización de las Naciones Unidas para la Educación, la Ciencia y la Cultura. Recuperado de http://unesdoc.unesco.org/images/0023/002354/235406e.pdf\#235452

Lemarchand, G. A. (2016). The scientific productivity and the dynamics of self-organizing networks: Iberoamerican and Caribbean countries (19662013). En M. Heitor, H. Horta \& J. Salmi (Eds.), Building capacity in Latin America: Trends and challenges in science and higher education (pp. 29-46). Nueva York: Springer.

Mehlum, H. M., Moene, K., \& Torvik, R. (2006). Institutions and the resource curse. Economic Journal, 116(508), 1-20. https://doi.org/10.1111/j.14680297.2006.01045.x

Organisation for Economic Cooperation and Development. (2010). Improving governance and measurement. En The OECD innovation strategy: Getting a head start on tomorrow (Cap. 7, pp. 189-213). Paris: Autor.

Organización de las Naciones Unidas para la Educación, la Ciencia y la Cultura. (2017). Relevamiento de la investigación y la Innovación en la República de Guatemala. En A. Lemarchand (Ed.), (Colección GOSPIN de perfiles nacionales en políticas de ciencia, tecnología e innovación, vol. 6). París: Autor.

Sachs, J. W., \& Warner, A. (1995). Natural Resource Abundance and Economic Growth. NBER Work- 
ing, (Paper No. 5398). https://doi.org/10.3386/ w5398

Sachs, J. W., \& Warner, A. (2001). The curse of natural resources. European Economic Review, 45(4-6), 827-838. https://doi.org/10.1016/S00142921(01)00125-8
Universidad del Valle de Guatemala, Instituto Nacional de Bosques, Consejo Nacional de Áreas Protegidas, \& Universidad Rafael Landívar. (2011). Mapa de cobertura forestal de Guatemala 2006 y dinámica de la cobertura forestal 2001-2006. Guatemala: Autor. 